\title{
The bHLH gene Hes1 is essential for expansion of early $T$ cell precursors
}

\author{
Koichi Tomita, ${ }^{1-3}$ Masakazu Hattori, ${ }^{3,4}$ Eijiro Nakamura, ${ }^{3}$ Shigetada Nakanishi, ${ }^{2}$ Nagahiro Minato, ${ }^{3}$ \\ and Ryoichiro Kageyama ${ }^{1,2,4}$
}

${ }^{1}$ Institute for Virus Research, Kyoto University, Kyoto 606-8507, Japan; Departments of ${ }^{2}$ Biological Sciences and
${ }^{3}$ Immunology and Cell Biology, Faculty of Medicine, Kyoto University, Kyoto 606-8501, Japan

Mice mutant for the bHLH gene Hes1, which is known to keep cells in a proliferative state, mostly lack thymus. Transfer of Hes1-null fetal liver cells into RAG2-null host mice normally reconstitutes B cells but fails to generate mature $\mathrm{T}$ cells in the thymus. In the reconstituted thymus, $\mathrm{T}$ cell differentiation is arrested at the $\mathrm{CD}^{-} \mathrm{CD8}^{-}$double negative (DN) stage. Both the initial $\mathrm{T}$ cell receptor (TCR)-independent and the subsequent TCR-dependent selective expansion during the DN stage are severely affected. Thus, Hes1 is essential for the earliest thymocyte expansion in a cell-autonomous manner.

[Key Words: bHLH; Hes1; T cell; thymocyte; thymus]

Received September 28, 1998; revised version accepted March 22, 1999.

T-cell precursors in the thymus are initially negative for expression of the surface antigens CD4 and CD8 [double negative (DN); Anderson et al. 1996; Shortman and Wu 1996; von Boehmer and Fehling 1997]. These DN cells then differentiate into $\mathrm{CD} 4^{+} \mathrm{CD} 8^{+}$[double positive (DP)] cells, which finally become $\mathrm{CD} 4^{+} \mathrm{CD} 8^{-}$or $\mathrm{CD} 4^{-} \mathrm{CD} 8^{+}$ mature single-positive (SP) cells. DN cells are subdivided further into four distinct differentiation stages based on changes in the surface expression of CD44 and CD25 in the following order: $\mathrm{CD} 44^{+} \mathrm{CD} 25^{-} \rightarrow \mathrm{CD} 44^{+} \mathrm{CD} 25^{+} \rightarrow$ $\mathrm{CD} 44^{-} \mathrm{CD} 25^{+} \rightarrow \mathrm{CD}_{4} 4^{-} \mathrm{CD} 25^{-}$(Godfrey and Zlotnik 1993; Godfrey et al. 1993, 1994; Pénit et al. 1995). At the first stage $\left(\mathrm{CD} 44^{+} \mathrm{CD} 25^{-}\right) \mathrm{DN}$ cells are resting and they start proliferation at the next stage $\left(\mathrm{CD} 44^{+} \mathrm{CD} 25^{+}\right)$. At the third stage $\left(\mathrm{CD} 44^{-} \mathrm{CD} 25^{+}\right)$, DN cells undergo rearrangement of $\mathrm{T}$ cell receptor (TCR) $\beta$ locus and, only when the $\beta$ locus is productively rearranged, these cells selectively proliferate and differentiate into DP cells. Thus, expansion of DN cells occurs at two distinct phases: the initial TCR-independent and the subsequent TCR-dependent selective proliferation (Pénit et al. 1995).

Although cell proliferation is very important for T-cell development because the majority of thymocytes eventually die after selection, the molecular mechanisms that control thymocyte proliferation are still largely unknown. The basic helix-loop-helix (bHLH) gene Hes1 (Sasai et al. 1992), which is expressed in the developing thymus from very early stages, is one of the candidate genes that regulate thymocyte proliferation, as persistent expression of Hes1 keeps cells in a proliferative

${ }^{4}$ Corresponding authors.

E-MAIL rkageyam@virus.kyoto-u.ac.jp; FAX 81-75-751-4807. E-MAIL hattori@med.kyoto-u.ac.jp; FAX 81-75-753-4403. state (Ishibashi et al. 1994; Tomita et al. 1996). Hes1 is a transcriptional repressor and functionally inhibits differentiation factors such as MyoD and Mash1 (Sasai et al. 1992; Ishibashi et al. 1995; Tomita et al. 1996; Chen et al. 1997). In the developing nervous system, Hes1 is expressed in dividing precursor cells, and only after Hes1 activity is lost do precursor cells stop cell division and start the differentiation program (Kageyama and Nakanishi 1997; Ström et al. 1997).

Here, we examined roles of Hes 1 in T cell development by reconstituting the lymphoid system of RAG2null mice with Hes1-null fetal liver cells. We found that $\mathrm{T}$ cell development is arrested at the DN stage in the absence of Hes1 and that Hes1 is essential for both the initial TCR-independent and the subsequent TCR-dependent selective expansion of thymocytes.

\section{Results and Discussion}

\section{Hes1 is essential for $T$ cell development}

Hes1 expression during normal thymic development was first determined by Northern blot analysis. Hes1 was already expressed at a high level in the developing thymus at embryonic day 15 (E15), but the expression gradually decreased afterwards (Fig. 1A). To determine the cell types that expressed Hes1, thymocytes and thymic stroma were separately prepared. Thymic stroma was prepared by treating the thymus with deoxyguanosine (dGUO) and it was negative for the thymocyte-specific CD3 expression (Fig. 1A). Hes1 was expressed in both thymocytes and thymic stroma (Fig. 1A), suggesting that Hes1 functions in both cell types.

To determine the Hes1 function in $\mathrm{T}$ cell develop- 


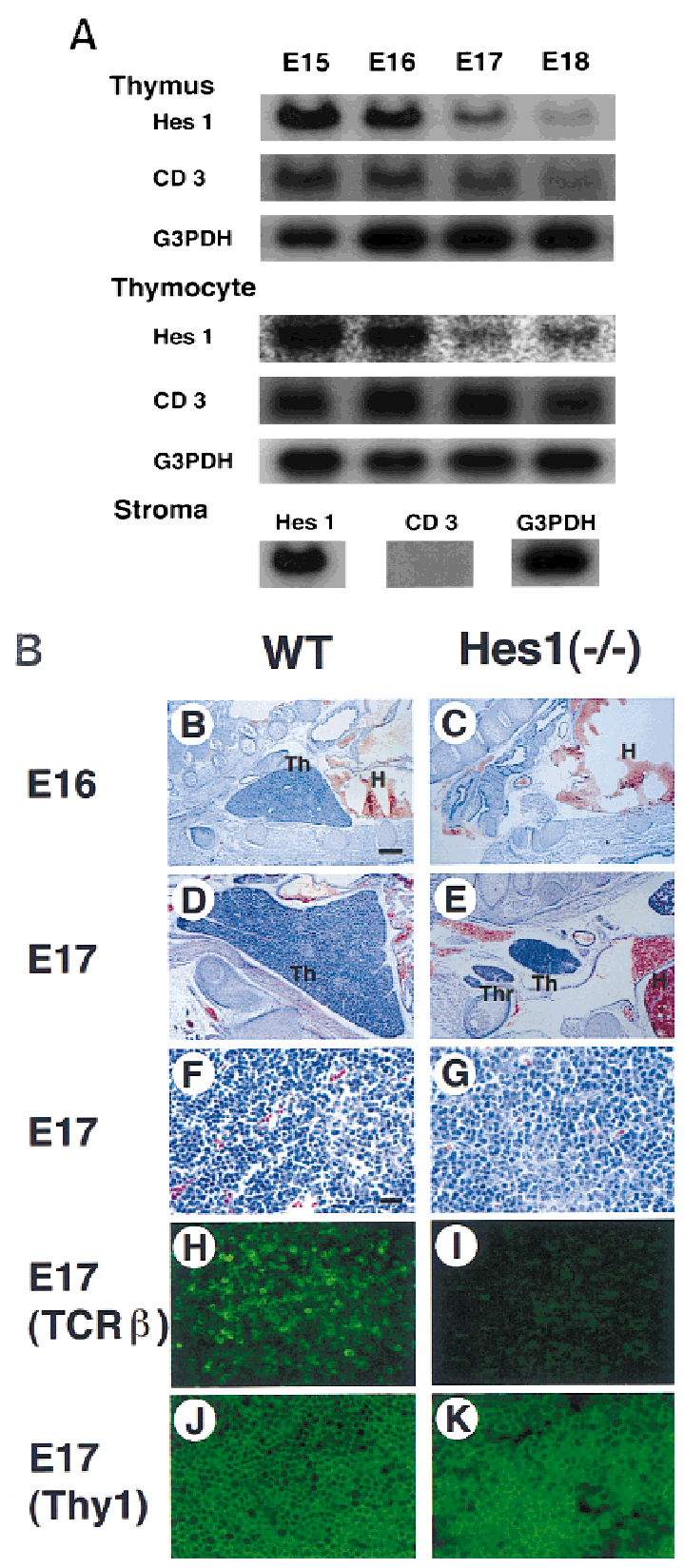

Figure 1. Hes1 expression in the developing thymus and the thymic defects of mice mutant for Hes1. (A) Hes1 expression in the thymus was determined by Northern blot analysis. Hes 1 was already expressed at a high level at E15, but expression decreased gradually afterward. Hes 1 was expressed by both thymocytes and stroma while CD3 was expressed only by thymocytes. G3PDH expression was examined as a control. $(B-K)$ Thymic sections were prepared from wild-type $(B, D, F, H, J)$ and Hes1-null $(C, E, G, I, K)$ embryos and subjected to HE staining $(B-$ $G)$ and immunohistochemistry with anti-TCR $\beta(H, I)$ and antiThyl antibodies $(J, K)$. Hes1-null embryos lacked thymus $(C)$ or had a very small thymus $(E)$ compared to wild type $(D) .(F, G)$ A higher magnification of $D$ and $E$. $(H, I)$ Even when the thymus existed, $\alpha \beta \mathrm{TCR}^{+}$thymocytes were absent in Hes1-null embryos $(I)$, whereas they were present in wild-type thymus $(H)$. $(J, K)$ In the Hes1-null thymus, there were Thy $1^{+}$cells $(K)$ as in the wild-type thymus $(J)$. Scale bar, $150 \mu \mathrm{m}(B-E) ; 25 \mu \mathrm{m}(F-K)$. (H) Heart; (Th) thymus; (Thr) thyroid. ment, we analyzed the mice mutant for Hes1. Hes1-null mice exhibit severe defects of neural tube and eye morphogenesis and die during gestation or just after birth (Ishibashi et al. 1995; Tomita et al. 1996). Surprisingly, $>90 \%$ of Hes1-null embryos completely lacked the thymus (Fig. 1C), and in the rest the thymus was much smaller in size than that of the wild type (Fig. 1D,E). In the thymus of Hes1-null mice, there were some thymocytes $\left(\right.$ Thy $1^{+}$, Fig. $\left.1 \mathrm{G}, \mathrm{K}\right)$ but TCR $\beta$ (Fig. 1I) and TCR $\gamma \delta$ expression (data not shown) was not detectable, suggesting that mature $\mathrm{T}$ cells were virtually absent. These results demonstrate that Hes1 is essential for T cell development.

Because Hes1 is also expressed in embryonal liver and spleen (Sasai et al. 1992), other lymphohematopoietic lineages in Hes1-null mice were examined by studying fetal liver cells. The number of the recovered Hes1-null fetal liver cells $\left[(2.0 \pm 0.4) \times 10^{7}\right]$ was comparable to that of the wild type cells $\left[(2.5 \pm 0.5) \times 10^{7}\right]$. Flow cytometric analysis revealed that erythroids (TER-119+, Fig. 2A), myeloids (Gr- $1^{+}$, Fig. $\left.2 \mathrm{~B}\right)$, and monocytes $\left(\mathrm{Mac}^{-}{ }^{+}\right.$, Fig. 2C) were normally generated in the absence of Hes1. In addition, Hes1-null fetal liver cells cultured on PA6 stromal cells in the presence of IL-7 (Sudo et al. 1989; Rolink et al. 1993) generated B-lineage cells comparably to wildtype cells $\left(\mathrm{IgM}^{+}, \mathrm{B} 220^{+}\right.$, Fig. 2D,E). Thus, cells of lymphohematopoietic lineages other than thymocytes appeared to develop normally in mice mutant for Hes1.

Hesl is critical for thymocyte expansion in a cell-autonomous manner

Because Hes1 is expressed in both stromal cells and thymocytes, the thymic phenotypes of Hes1-null mice could be due to abnormality of stromal cells and/or thymocytes. To determine whether Hes1-null thymocytes are intrinsically affected, we made bone marrow chimeras using RAG2-null mutant mice, which have no mature T or B lymphocytes (Shinkai et al. 1992). Fetal liver cells from Hes1-null or wild-type donor embryos were injected intravenously into irradiated RAG2-null mice of C57BL/6 background to allow differentiation of donor progenitors in the environment of normal thymic stromal cells. Lymphoid cells $\left(\mathrm{Thy}^{+}{ }^{+}\right)$of the donor origin were distinguished by Ly5.2 expression from those of possible host origin that are negative for Ly5.2 (Fig. 3B). This analysis could also help determine more precisely the stage at which $\mathrm{T}$ cell development is impaired in the absence of Hes1.

When reconstituted with wild-type or Hes1-null fetal liver cells, the RAG2-null hosts had a comparable number of mature B cells in the spleen $\left(\mathrm{IgM}^{+}, \mathrm{B} 220^{+}\right.$, Fig. $3 \mathrm{C}, \mathrm{D})$, indicating that reconstitution was successful and that B cells develop normally in the absence of Hes1. In contrast, the absolute number of thymocytes of the Hes1-null origin was by far less than that of the wildtype origin in the reconstituted RAG2-null thymus (Fig. $3 \mathrm{~A})$. When $1 \times 10^{6}$ and $1 \times 10^{7}$ fetal liver cells of wildtype origin were injected, an average of $1.6 \times 10^{8}(n=13)$ and $1.0 \times 10^{8}(n=6)$ thymocytes, respectively, was re- 

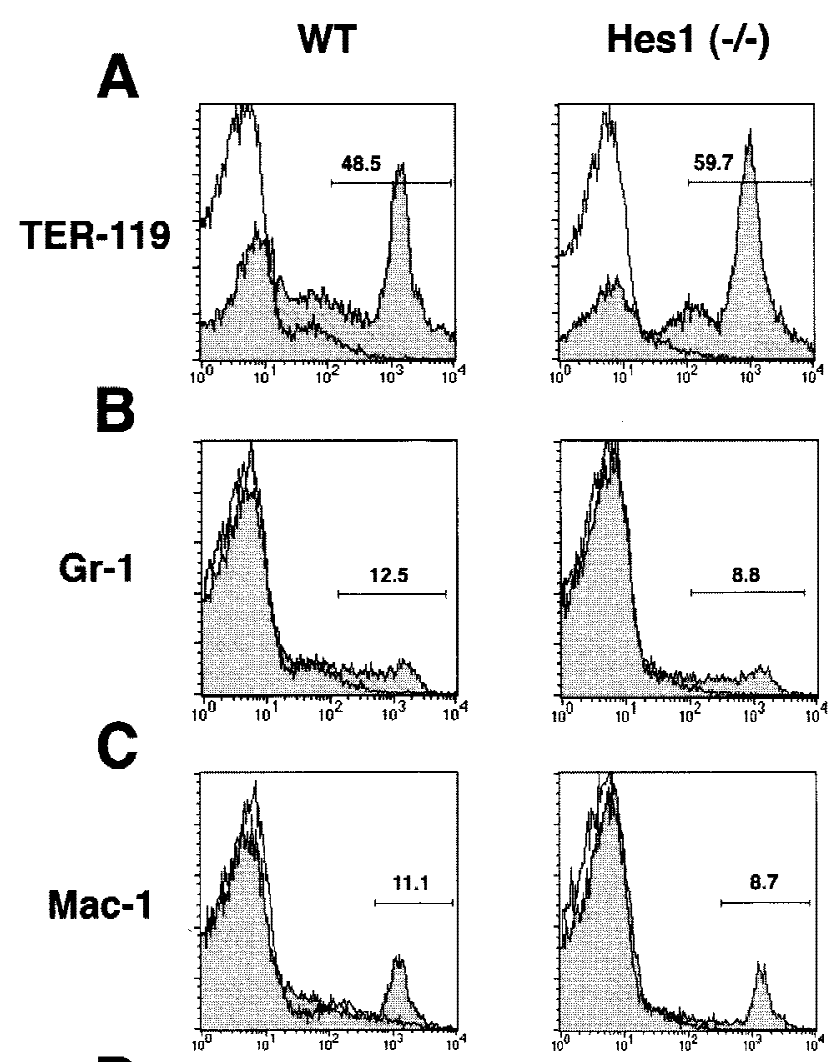

D
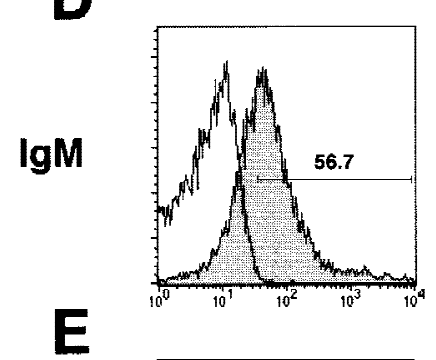

B220
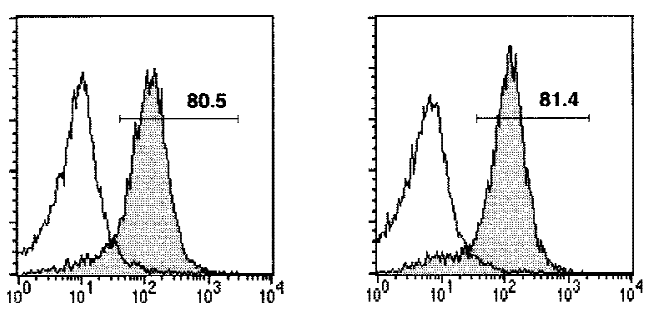

Figure 2. Flow cytometric analysis of fetal liver cells. Flow cytometric analysis was performed to determine the surface expression of TER-119 $(A)$, Gr-1 $(B)$, and Mac-1 $(C)$. For B cell lineage analysis, fetal liver cells were cultured on PA6 stromal cells in the presence of IL-7 and examined for expression of IgM $(D)$ and B220 $(E)$. Cells were analyzed after incubation in the presence (shaded area) or absence (bold line only) of FITC-conjugated antibodies. No abnormality was detected in Hes1-null cells.

covered. In contrast, when $1 \times 10^{6}$ Hes1-null cells were injected, only $1.7 \times 10^{5}$ thymocytes were recovered $(n=9)$; and even when $1 \times 10^{7}$ Hes 1 -null cells were injected, only $4.1 \times 10^{5}$ thymocytes were recovered $(n=8)$, indicating that the thymocyte number of the Hes1-null origin was 200- to 1000-fold less than that of wild-type origin. The thymocyte number of the Hes1-null origin was still 10- to 20-fold less than that of the nonreconstituted RAG2-null host mice $\left(3.2 \times 10^{6}\right.$ cells; $\left.n=10\right)$ (Fig. $3 \mathrm{~A})$. These results demonstrate that thymocytes are intrinsically affected by Hes1 mutation and that Hes1 is critical for expansion of thymocytes.

\section{Thymocyte expansion at the DN stage is impaired by Hes1 mutation}

To determine the stage at which $\mathrm{T}$ cell development is impaired in the absence of Hes1, thymocytes of the donor origin were analyzed further with various surface markers. Whereas both $\alpha \beta$ and $\gamma \delta$ T cells were generated from wild-type donors, neither mature $\mathrm{T}$ cells were detected in the thymus reconstituted with Hes1-null donor cells (Fig. 4A). Furthermore, the vast majority of thymocytes of Hes1-null origin were negative for expression of the surface antigens CD4 and CD8 (Fig. 4B) but highly expressed the early marker heat-stable antigen (HSA) in the virtual absence of CD3 (Fig. 4C). Thus, in the absence of Hes1, T cell development is arrested at the $\mathrm{CD}^{-} \mathrm{DN}$ stage. Essentially the same abnormality was observed irrespective of the number of the injected cells $\left(1 \times 10^{6}\right.$ or $1 \times 10^{7}$, Fig. $\left.4 \mathrm{~A}-\mathrm{C}\right)$.

Because DN cells are further divided into four distinct differentiation stages based on the profiles in the surface expression of CD44 and CD25 (Godfrey and Zlotnik 1993; Godfrey et al. 1993, 1994; Pénit et al. 1995), donor DN thymocytes were analyzed with these markers (Fig. $4 \mathrm{D})$. At the first stage $\left(\mathrm{CD} 44^{+} \mathrm{CD} 25^{-}\right) \mathrm{DN}$ cells are normally resting and the absolute number of Hes1-null DN thymocytes was, on an average, $1.3 \times 10^{5}$ cells $(n=4)$ and $0.6 \times 10^{5}$ cells $(n=4)$ when $1 \times 10^{6}$ and $1 \times 10^{7}$ cells, respectively, were injected. These numbers were comparable to those of wild-type DN cells $\left(1.2 \times 10^{5}\right.$ cells; $n=9$ ). However, when $1 \times 10^{6}$ Hes 1 -null cells were injected, at the subsequent stages the number of Hes1-null thymocytes was by far less than that of the wild-type cells, which are known to proliferate TCR independently first and then TCR dependently during the DN stages (Fig. 4D). When $1 \times 10^{7}$ Hes1-null cells were injected, DN thymocytes increased at the $\mathrm{CD} 44^{-} \mathrm{CD} 25^{+}$stage $\left(2.2 \times 10^{5}\right.$ cells; $\left.n=4\right)$, but the number was still much less than the wild type $\left(1.5 \times 10^{6}\right.$ cells; $\left.n=9\right)($ Fig. $4 \mathrm{D})$. Thus, proper expansion of DN thymocytes was severely impaired in the absence of Hes1.

The spleen reconstituted with Hes1-null donors contained virtually no $\alpha \beta \mathrm{T}$ cells, but interestingly it had some $\gamma \delta \mathrm{T}$ cells (Fig. 4E). Because the RAG2-null host mice did not have such $\mathrm{T}$ cells (Fig. 4F), they were derived from Hes1-null fetal liver cells. Thus, some $\gamma \delta \mathrm{T}$ cells may develop normally in the absence of Hes1, although it remains to be determined whether they differentiate at the intrathymic or extrathymic environments.

\section{Both TCR-independent and-dependent thymocyte} expansion is affected by Hes1 mutation

DN cells normally undergo rearrangement of TCR $\beta$ lo- 
Figure 3. Reconstitution of the lymphoid system of $R A G 2$ mutant mice by transfer of wild-type and Hes1-null fetal liver cells. Either wild-type or Hes1-null fetal liver cells $\left(1 \times 10^{6}\right.$ or $1 \times 10^{7}$, indicated in parentheses above the panel) were transferred intravenously into $R A G 2$ mutant mice to reconstitute the lymphoid system, and after 4-6 weeks of transfer, the lymphoid cells were examined. (A) The absolute numbers of total thymocytes. From the reconstituted thymuses, only Ly $5.2^{+}$cells were counted. The average thymocyte number with standard error is $R A G 2^{-1-}$, $(3.2 \pm 0.6) \times 10^{6} \quad(n=10) ; \quad$ RAG $2^{-/-}{ }^{\prime}$ $\mathrm{WT}\left(1 \times 10^{6}\right.$ cells injected), $(1.6 \pm 0.3) \times$ $10^{8} \quad(n=13) ; \quad R A G 2^{-/-} / \mathrm{WT}\left(1 \times 10^{7}\right.$ cells injected), $(1.0 \pm 0.2) \times 10^{8} \quad(n=6)$; $R A G 2^{-1-} /$ Hes $^{-1-}\left(1 \times 10^{6}\right.$ cells injected $)$, $(1.7 \pm 0.7) \times 10^{5} \quad(n=9) ; \quad$ RAG2 $2^{-/-} /$Hes $^{-1}$ $-\left(1 \times 10^{7}\right.$ cells injected $),(4.1 \pm 1.0) \times 10^{5}$ $(n=8)$. The number of Hes1-null thymocytes averaged 200- to 1000 -fold less than that of wild-type thymocytes and 10- to 20 -fold less than that of irradiated and nonreconstituted $R A G 2$-null thymocytes. (B) Flow cytometric analysis of the reconstituted thymus with $\alpha$-Ly5.2 and $\alpha$-Thy1. The absolute number of total cells is indicated above each panel; the number of

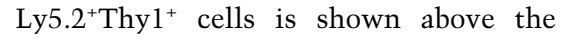
gated region. $(C)$ The absolute numbers of $\mathrm{B}$ cells in the spleen. The B cell number of Hes1-null origin was normal. (D) Flow cytometric analysis of the reconstituted spleen with $\alpha$-IgM and $\alpha$-B220. B cells developed normally in the absence of Hes1.
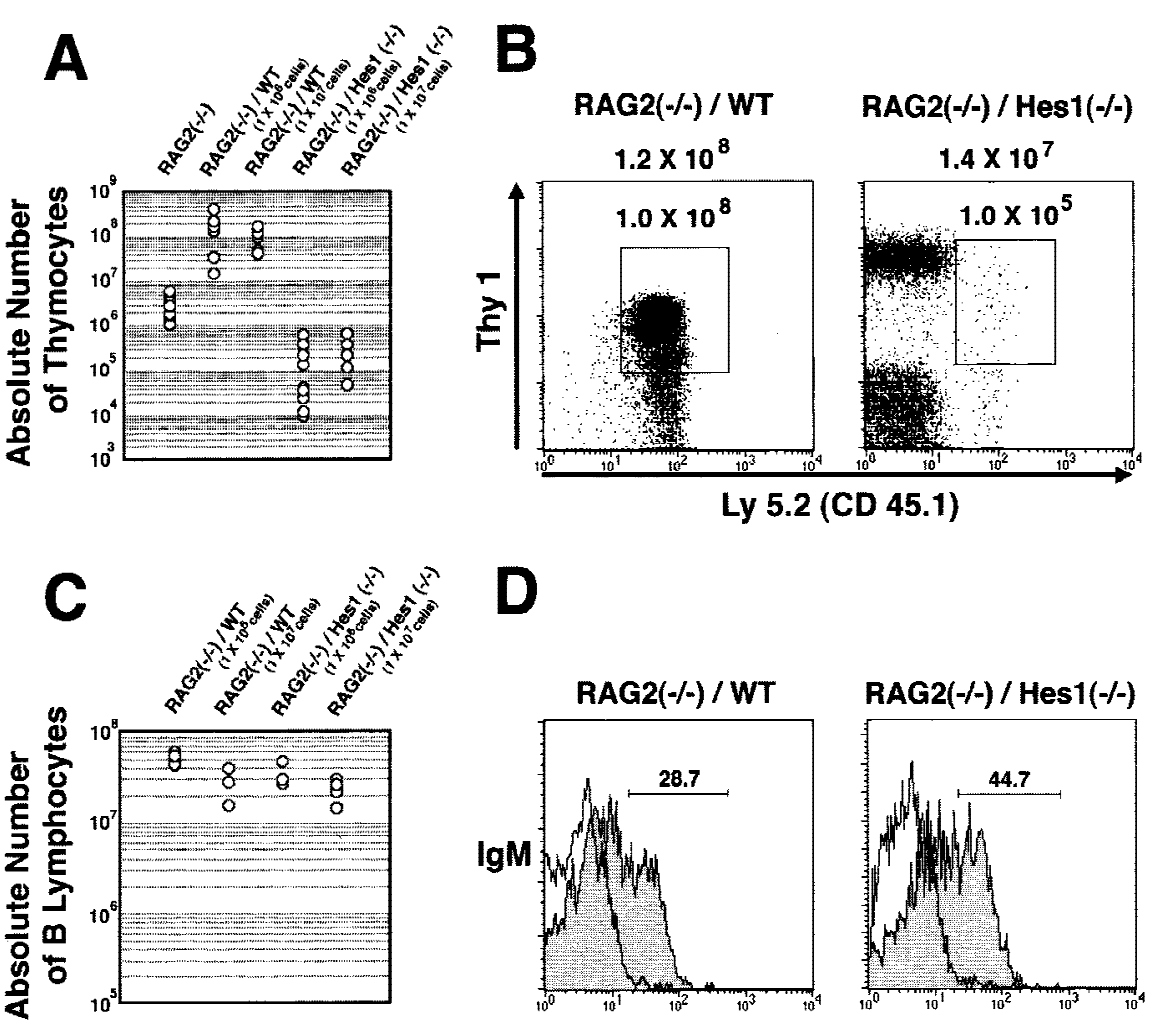

D
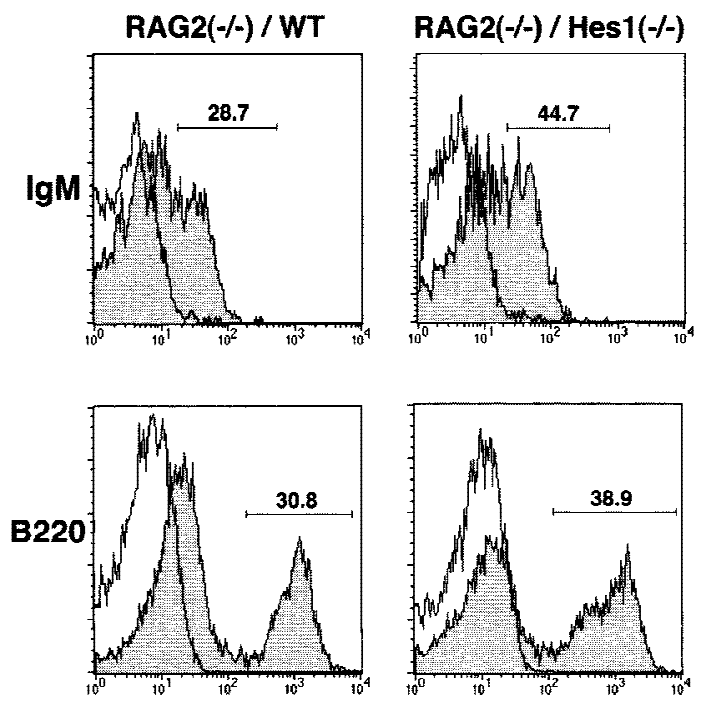

cus at the $\mathrm{CD} 44^{-} \mathrm{CD} 25^{+}$stage, and only when the $\beta$ locus is productively rearranged are these cells allowed to selectively proliferate and mature into the last stage (CD44-CD25-) (Godfrey and Zlotnik 1993; Godfrey et al. 1993, 1994; Pénit et al. 1995). Because the thymocyte number at the $\mathrm{CD} 44^{-} \mathrm{CD} 25^{+}$and $\mathrm{CD} 44^{-} \mathrm{CD} 25^{-}$stages was reduced significantly by Hes 1 mutation, it is possible that TCR gene rearrangement is affected in Hes1null thymocytes. We therefore examined rearrangement of TCR genes by amplifying the genomic DNA of the reconstituted thymi (Anderson et al. 1992; Itohara et al. 1993; Maki et al. 1996). Because in reconstituted mice Hes1-null thymocytes were, on an average, 200- to 1000fold less in number than wild-type thymocytes, we used a 500-fold dilution of the wild-type DNA as a template to normalize the cell numbers. In the thymus and spleen of RAG2 mutant mice reconstituted with wild-type cells, TCR gene rearrangements clearly occurred $1+/+$ in Fig. $5 \mathrm{~A}-\mathrm{H})$. In contrast, when $R A G 2$ mutant mice were reconstituted with $1 \times 10^{6}$ Hes1-null cells, complete rearrangement was not detected at the TCR $\alpha$ or $\beta$ locus (-/in Fig. 5A,E), although only a low level of $D-J$ recombi- nation could be detected at the TCR $\beta$ locus $(-/-$ in Fig. $5 \mathrm{C})$. TCR $\gamma$ gene rearrangement was not detected either in the thymus reconstituted with $1 \times 10^{6}$ Hes1-null cells (-/- in Fig. 5G). These results demonstrated that the Hes1 mutation severely affected expansion of immature thymocytes before the initiation of TCR gene rearrangement. However, when RAG2 mutant mice were reconstituted with $1 \times 10^{7}$ Hes1-null cells, TCR gene rearrangements became detectable $(-/-$ in Fig. 5B,D,F,H), suggesting that Hes1 is not a prerequisite for the TCR gene rearrangements per se. Even when TCR gene rearrangements could take place, Hes1-null thymocytes did not expand properly at the $\mathrm{CD} 44^{-} \mathrm{CD} 25^{+}$and CD $44^{-}$CD $25^{-}$stages (see above), suggesting that TCRdependent selective proliferation seemed to be disturbed in the absence of Hes1. However, the amount of TCR gene rearrangements was still low in the absence of Hes1; therefore, future experiments with TCR transgenic in the Hes1-/- background will be required to determine the role of Hes1 in TCR-dependent expansion and differentiation.

Interestingly, TCR $\gamma 2$ and TCR $\gamma 4$ gene rearrangements 


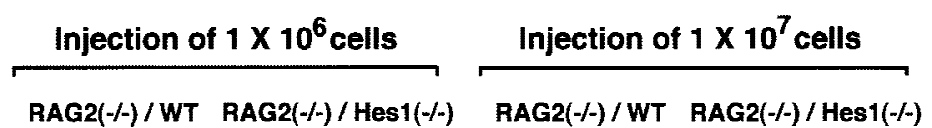

A

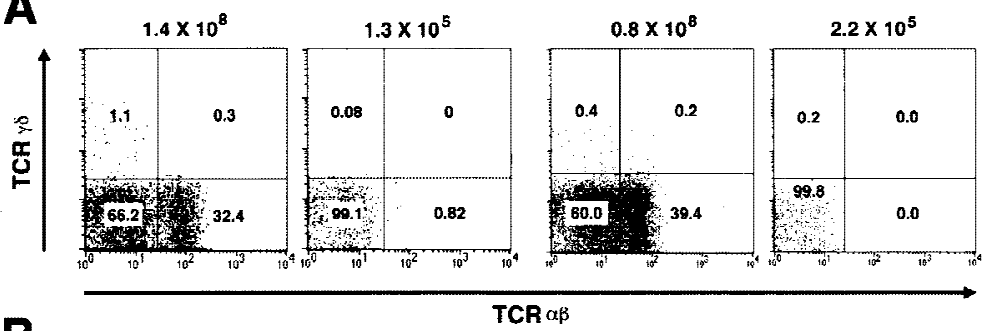

B

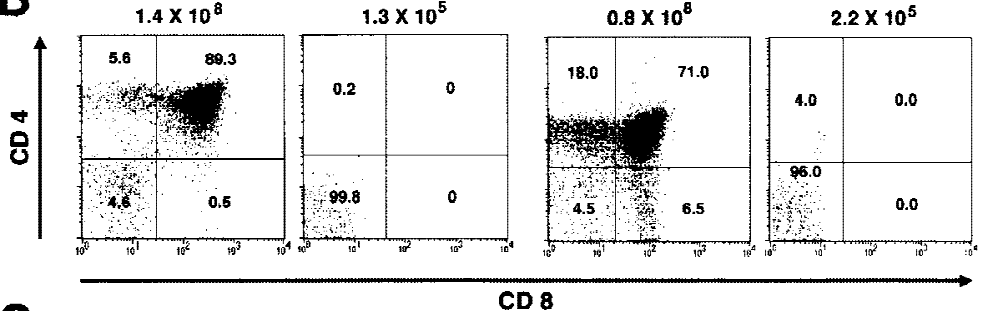

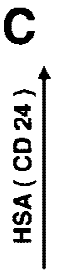
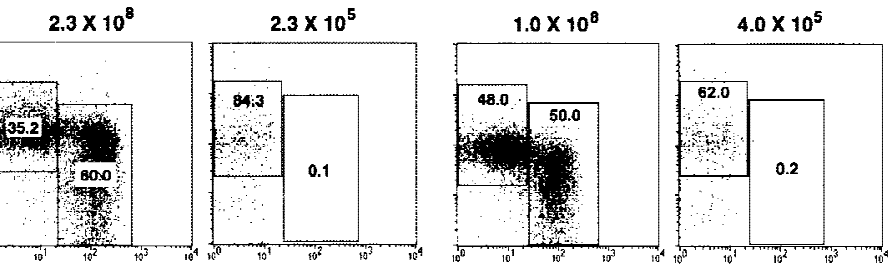

D

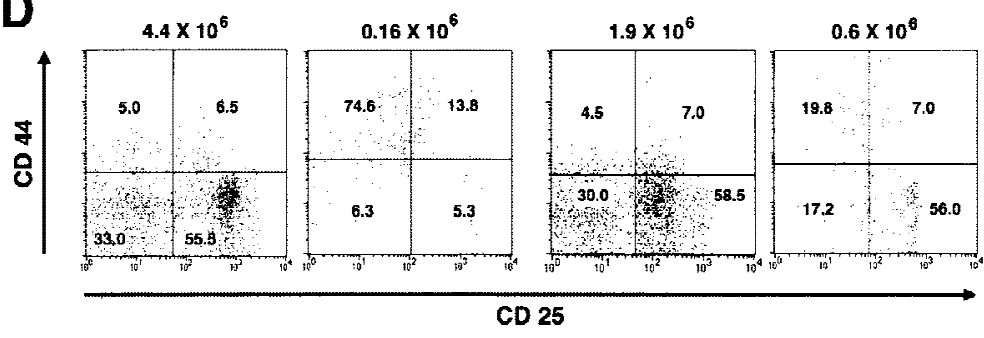

E

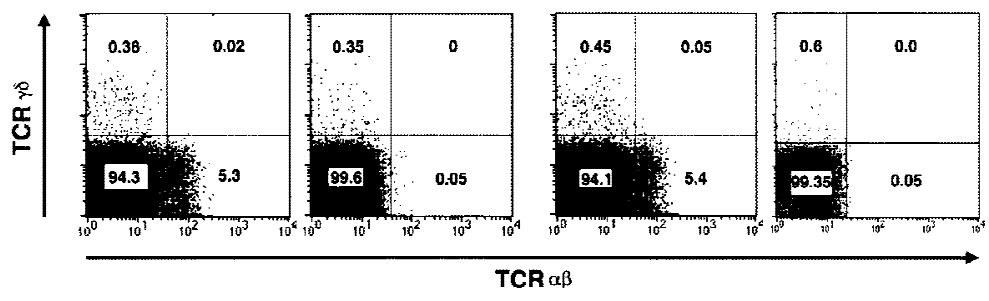

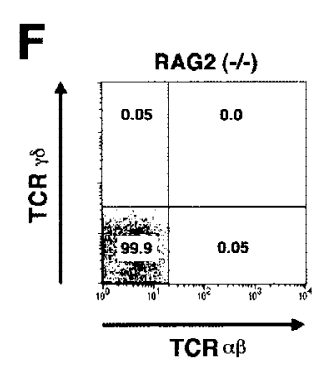

Figure 4. Flow cytometric analysis of reconstituted T-lineage cells. Flow cytometric analysis of reconstituted thymocytes $(A-D)$ and spleen cells $(E)$ and nonreconstituted host spleen cells $(F)$ was performed to determine the surface expression of $\alpha \beta$ and $\gamma \delta$ TCR $(A, E, F)$, CD4 and CD8 $(B)$, HSA and CD3 $(C)$, and CD44 and CD25 $(D)$. The absolute number of Ly5.2+ thymocytes is indicated above each panel $(A-$ $C)$. For analysis of surface expression of CD44 and CD25, DN thymocytes from donor origin were analyzed $(D)$, and the number is shown above each panel $(D)$. In the absence of Hes1, $\alpha \beta$ and $\gamma \delta \mathrm{TCR}^{+}$cells, $\mathrm{CD} 4^{+} \mathrm{CD} 8^{+} \mathrm{DP}$ cells, and $\mathrm{CD} 3^{+}$cells were virtually absent in the thymus $(A-C)$. In the spleen reconstituted with Hes1-null cells, $\gamma \delta$ T cells were present at a comparable level to the wild type, whereas $\alpha \beta \mathrm{T}$ cells were not detected $(E)$. were consistently and selectively detectable in the spleen but not in the thymus when RAG2 mutant mice were reconstituted with $1 \times 10^{6}$ Hes1-null fetal liver cells $(-/-$ in Fig. 5G). Thus, $\gamma \delta \mathrm{T}$ cells with restricted repertoire could develop normally in the absence of Hes1.

\section{Unique and essential functions of Hes1}

in $T$ cell development

In this study, we have demonstrated that early events of $\alpha \beta$ and $\gamma \delta$ T cell development in the thymus, such as cell expansion and TCR gene rearrangement, are severely impaired in Hes1-null mice. Particularly, Hes1 seems to be essential for cell expansion at the very early stages, as Hes1-null cells did not expand at the CD $44^{+} \mathrm{CD} 25^{+}$stage in the reconstituted thymus when $1 \times 10^{6}$ fetal liver cells were injected. Homing of Hes1-null progenitors to the thymus appeared unaffected, as the number of Hes1-null thymocytes at the $\mathrm{CD} 44^{+} \mathrm{CD} 25^{-}$stage was comparable with that of wild-type cells. It is likely that Hes1-null thymocytes might remain as resting cells or be more 

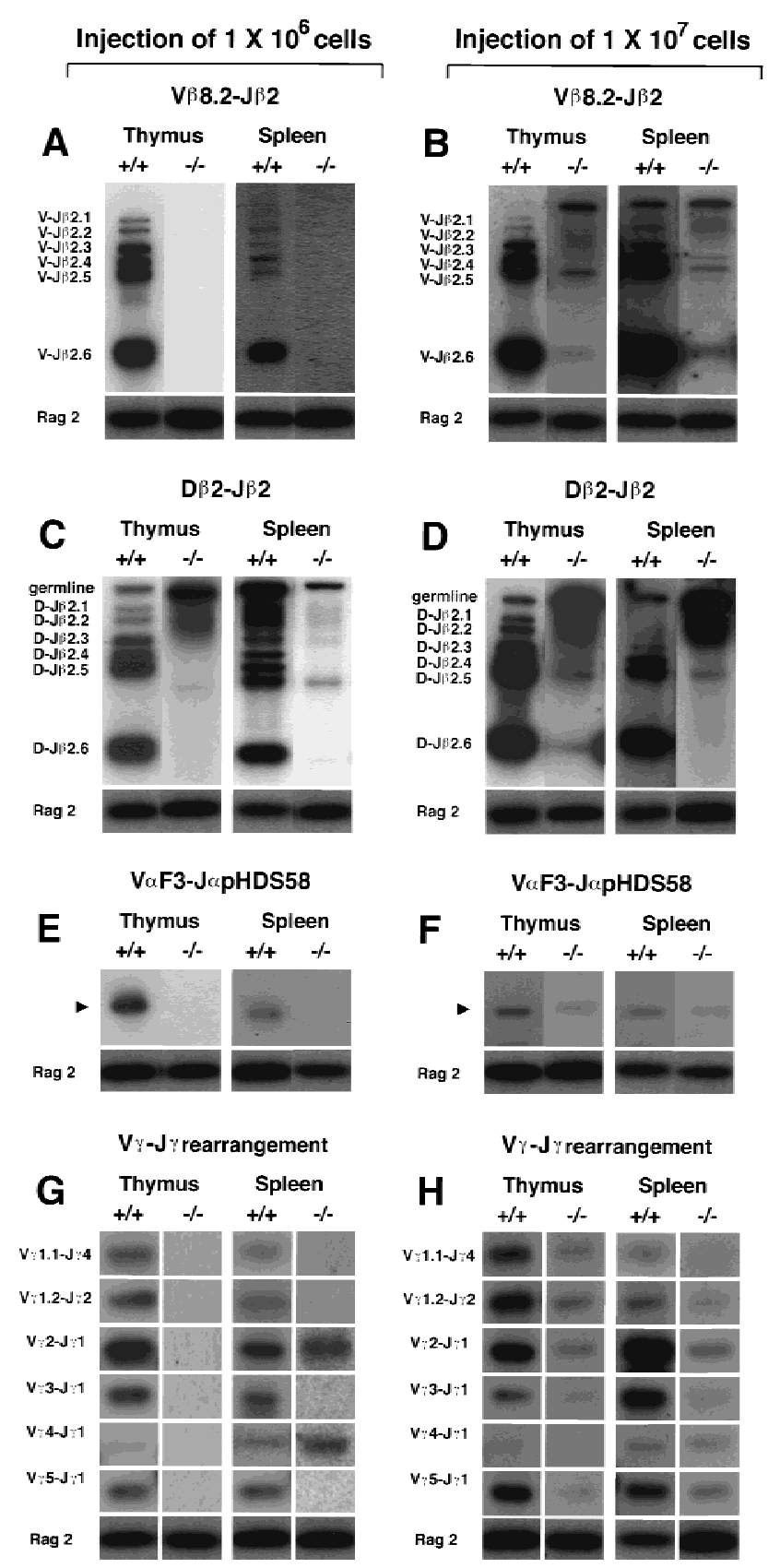

Figure 5. Rearrangement of TCR genes. Either $1 \times 10^{6}$ $(A, C, E, G)$ or $1 \times 10^{7}(B, D, F, H)$ fetal liver cells of wild-type $(+/+)$ and Hes1-null origin $(-/-)$ were transferred intravenously into RAG2 mutant mice, and rearrangements of TCR genes were examined by PCR followed by Southern blot analysis with ${ }^{32} \mathrm{P}$ labeled oligonucleotide probes. In the thymus reconstituted with $1 \times 10^{6}$ Hes1-null cells, no apparent rearrangement was detected in the TCR $\alpha$ and TCR $\beta$ loci $(A, E)$, although only a low level of $D-J$ recombination could be detected $(C)$. Whereas TCR $\gamma$ gene rearrangement was not detected in the thymus reconstituted with $1 \times 10^{6}$ Hes1-null cells, TCR $\gamma 2$ and TCR $\gamma 4$ genes (Maki et al. 1996) were rearranged in the spleen in the absence of Hes1 $(G)$. When $1 \times 10^{7}$ Hes1-null cells were injected, TCR gene rearrangement was detected $(B, D, F, H)$. As an internal control for PCR, the RAG2 gene was amplified. The RAG2 signal was derived only from the donor cells. susceptible to apoptosis. This defect of expansion might contribute to the lack of or a small-sized thymus in Hes1 mutant mice, although it is possible that certain functional defects of the Hes1-null stromal cells additionally contribute to the thymic aplasia.

It has been shown that the selective proliferation of DN cells that express functional TCRs involves various transcription factors such as HMG-box factors LEF-1 and TCF-1 and zinc finger transcription factor GATA-3 (Ting et al. 1996; Okamura et al. 1998). However, none of these factors appears to be involved in cell expansion before TCR gene rearrangement. Thus, Hes1 is unique in regulating TCR-independent expansion of thymocytes at the earliest stage. Interestingly, in the absence of IL-7 receptor, thymocyte expansion is severely disturbed before TCR gene rearrangement (Peschon et al. 1994; Candéias et al. 1997), suggesting that IL-7 is one of the major growth factors involved in initial TCR-independent thymocyte proliferation. It is therefore tempting to speculate that Hes1 functions downstream of the IL-7 signaling pathway. In addition to TCR-independent expansion, Hes1 seems to be involved in expansion at later stages, as-even though TCR gene rearrangement occurred in the thymus of RAG2-null mice reconstituted with as many as $1 \times 10^{7}$ fetal liver cells-Hes1-null thymocytes were still confined exclusively to the DN fraction with negligible increase in cell number. In the developing nervous system, Hes1 is known to keep cells in a proliferative state and down regulation of Hes1 expression leads to transition to a nonproliferative differentiation stage (Kageyama and Nakanishi 1997). Present results have indicated that similarly in the thymus Hes 1 promotes the initial expansion of immigrant progenitor cells, which is essential for the extensive clonal diversification and selection to generate mature $\mathrm{T}$ cells.

Hes1 is also known as a target gene of the Notch signaling (Jarriault et al. 1995; Nishimura et al. 1998). It has been shown that both Notch and Hes1 regulate CD4 SP versus CD8 SP fate choice of T cell development (Robey et al. 1996; Kim and Siu 1998). Although this function could not be examined here, as T cell development was arrested much earlier by Hes 1 mutation, collectively these data suggest that Hes1 might function at multiple steps of $\mathrm{T}$ cell development.

\section{Materials and methods}

\section{Hes 1 expression in the thymus}

Northern blot analysis was done as described previously (Sasai et al. 1992). The thymic stroma devoid of thymocytes was prepared as reported (Ikuta et al. 1990), with some modifications: Fetal thymic lobes (E15) were cultured at $37^{\circ} \mathrm{C}$ for 6 days in $7 \%$ $\mathrm{CO}_{2}$ on filters in 12-well plates containing RPMI1640 supplemented with $10 \%$ fetal calf serum, $1.35 \mathrm{~mm}$ dGUO, and $50 \mu \mathrm{M}$ $\beta$-mercaptoethanol.

\section{Histological analysis of mouse embryos}

Whole embryos were fixed with $4 \%$ paraformaldehyde in PBS at $4^{\circ} \mathrm{C}$ for $1 \mathrm{hr}$ and incubated in $30 \%$ sucrose in PBS at $4^{\circ} \mathrm{C}$ over- 
night. Frozen sections were prepared at $16 \mu \mathrm{m}$ thickness and subjected to hematoxylin-eosin (HE) staining and immunohistochemistry. For immunohistochemistry, sections were incubated with biotinylated anti-TCR $\beta$ (Pharmingen, H57-597) and anti-Thyl antibody (Phamingen, 53-2.1) followed by visualization with avidin-labeled fluorescein (Vector).

\section{Fetal liver cell analysis}

Single cell suspensions from fetal livers of E17 wild-type or Hes1-null embryos were prepared and subjected to flow cytometric analysis (Becton-Dickinson). The antibodies used were as follows: anti-TER-119 (Pharmingen, TER-119), anti-Gr-1 (Pharmingen, RB6-8C5), and anti-Mac-1 (Pharmingen, M1/70). Cultured fetal liver cells on PA6 stromal cells with IL-7 were subjected to immunostaining with anti-IgM (Pharmingen, R660.2), and anti-B220 antibodies (Pharmingen, RA3-6B2). Detailed culture conditions were described previously (Sudo et al. 1989; Rolink et al. 1993).

\section{Reconstitution of RAG2 mutant mice}

Fetal liver cells $\left(1 \times 10^{6}\right.$ to $\left.10 \times 10^{6}\right)$ from E13-E16 wild-type and Hes1-null embryos (Ly5.2 $2^{+}$were injected intravenously into 4 Gy irradiated RAG2 mutant mice (Ly5.2-). After 4-6 weeks, single cell suspensions were prepared from thymuses and spleens of the reconstituted RAG2 mutant mice and subjected to flow cytometric analysis (Becton-Dickinson). Only Ly5.2 ${ }^{+}$ cells (the donor origin) were analyzed. The antibodies used were as follows: anti-TCR $\beta$ (Pharmingen, H57-597), anti- $\gamma \delta$ TCR (Pharmingen, GL-3), anti-CD4 (Pharmingen, RM4-5), anti-CD8 (Pharmingen, 53-6.7), anti-CD25 (Pharmingen, 7D4), anti-CD44 (Pharmingen, IM7), anti-CD24 (HSA) (Pharmingen, M1/69), anti-CD3 (Pharmingen, 145-2C11), anti-Thy1 (Phamingen, 532.1), and anti-CD45.1 (Ly5.2) (Pharmingen, A20).

\section{PCR analysis for TCR gene rearrangement}

The genomic DNA extracted from the reconstituted thymuses was amplified by PCR and subjected to Southern blot analysis with ${ }^{32}$ P-labeled oligonucleotide probes as described previously (Anderson et al. 1992; Itohara et al. 1993; Maki et al. 1996). Because the numbers of Hes1-null thymocytes were 200- to 1000 -fold less than those of wild-type thymocytes, we used a 500 -fold dilution of the wild-type samples to normalize the cell numbers. As a PCR control, we amplified the RAG2 gene, which was derived only from donor cells. In each experiment, a similar intensity of the RAG2 band was detected, indicating that the number of donor cells was normalized.

\section{Acknowledgments}

We thank Koichi Ikuta for RAG2 mutant mice and discussion, Kazuhiro Iwai, Shunichi Takeda, Yoshimoto Katsura and Kazushige Maki for discussion, and Tetsuo Sudo for IL-7. This work was supported by Special Coordination Funds for Promoting Science and Technology and research grants from the Ministry of Education, Science, Sports, and Culture of Japan and from Japan Society for the Promotion of Science.

The publication costs of this article were defrayed in part by payment of page charges. This article must therefore be hereby marked 'advertisement' in accordance with 18 USC section 1734 solely to indicate this fact.

\section{References}

Anderson, G., N.C. Moore, J.J.T. Owen, and E.J. Jenkinson.
1996. Cellular interactions in thymocyte development. Annu. Rev. Immunol. 14: 73-99.

Anderson, S.J., K.M. Abraham, T. Nakayama, A. Singer, and R.M. Perlmutter. 1992. Inhibition of T-cell receptor $\beta$-chain gene rearrangement by overexpression of the non-receptor protein tyrosine kinase p56 ${ }^{\text {lck }}$. EMBO J. 11: 4877-4886.

Candéias, S., K. Muegge, and S.K. Durum. 1997. IL-7 receptor and VDJ recombination: Trophic versus mechanistic actions. Immunity 6: 501-508.

Chen, H., A. Thiagalingam, H. Chopra, M.W. Borges, J.N. Feder, B.D. Nelkin, S.B. Baylin, and D.W. Ball. 1997. Conservation of the Drosophila lateral inhibition pathway in human lung cancer: A hairy-related protein (HES-1) directly represses achaete-scute homolog-1 expression. Proc. Natl. Acad. Sci. 94: 5355-5360.

Godfrey, D.I. and A. Zlotnik. 1993. Control points in early Tcell development. Immunol. Today 14: 547-553.

Godfrey, D.I., J. Kennedy, T. Suda, and A. Zlotnik. 1993. A developmental pathway involving four phenotypically and functionally distinct subsets of $\mathrm{CD}^{-} \mathrm{CD}^{-} \mathrm{CD}^{-}$triplenegative adult mouse thymocytes defined by CD44 and CD25 expression. J. Immunol. 150: 4244-4252.

Godfrey, D.I., J. Kennedy, P. Mombaerts, S. Tonegawa, and A. Zlotnik. 1994. Onset of TCR- $\beta$ gene rearrangement and role of TCR- $\beta$ expression during $\mathrm{CD}^{-} \mathrm{CD}^{-} \mathrm{CD}^{-}$thymocyte differentiation. J. Immunol. 152: 4783-4792.

Ikuta, K., T. Kina, I. MacNeil, N. Uchida, B. Peault, Y. Chien, and I.L. Weissman. 1990. A developmental switch in thymic lymphocyte maturation potential occurs at the level of hematopoietic stem cells. Cell 62: 863-874.

Ishibashi, M., K. Moriyoshi, Y. Sasai, K. Shiota, S. Nakanishi, and R. Kageyama. 1994. Persistent expression of helix-loophelix factor HES1 prevents mammalian neural differentiation in the central nervous system. EMBO J. 13: 1799-1805.

Ishibashi, M., S.-L. Ang, K. Shiota, S. Nakanishi, R. Kageyama, and F. Guillemot. 1995. Targeted disruption of mammalian hairy and Enhancer of split homolog-1 (HES-1) leads to upregulation of neural helix-loop-helix factors, premature neurogenesis, and severe neural tube defects. Genes \& Dev. 9: 3136-3148.

Itohara, S., P. Mombaerts, J. Lafaille, J. Iacomini, A. Nelson, A.R. Clarke, M.L. Hopper, A. Farr, and S. Tonegawa. 1993. T cell receptor $\delta$ gene mutant mice: Independent generation of $\alpha \beta$ T cells and programmed rearrangement of $\gamma \delta$ TCR genes. Cell 72: 337-348.

Jarriault, S., C. Brou, F. Logeat, E.H. Schroeter, R. Kopan, and A. Israel. 1995. Signalling downstream of activated mammalian Notch. Nature 377: 355-358.

Kageyama, R. and S. Nakanishi. 1997. Helix-loop-helix factors in growth and differentiation of the vertebrate nervous system. Curr. Opin. Genet. Dev. 7: 659-665.

Kim, H.K. and G. Siu. 1998. The Notch pathway intermediate HES-1 silences CD4 gene expression. Mol. Cell. Biol. 18: 7166-7175.

Maki, K., S. Sunaga, and K. Ikuta. 1996. The V-J recombination of $\mathrm{T}$ cell receptor- $\gamma$ genes is blocked in interleukin-7 receptor-deficient mice. J. Exp. Med. 184: 2423-2427.

Nishimura, M., F. Isaka, M. Ishibashi, K. Tomita, H. Tsuda, S. Nakanishi, and R. Kageyama. 1998. Structure, chromosomal locus, and promoter of mouse Hes2 gene, a homologue of Drosophila hairy and Enhancer of split. Genomics 49: 6975.

Okamura, R.M., M. Sigvardsson, J. Galceran, S. Verbeek, H. Clevers, and R. Grosschedl. 1998. Redundant regulation of T cell differentiation and TCR $\alpha$ gene expression by the transcription factors LEF-1 and TCF-1. Immunity 8: 11-20. 
Pénit, C., B. Lucas, and F. Vasseur. 1995. Cell expansion and growth arrest phases during the transition from precursor $\left(\mathrm{CD}^{-} 8^{-}\right)$to immature $\left(\mathrm{CD}^{+} 8^{+}\right)$thymocytes in normal and genetically modified mice. J. Immunol. 154: 5103-5113.

Peschon, J.J., P.J. Morrissey, K.H. Grabstein, F.J. Ramsdell, E. Maraskovsky, B.C. Gliniak, L.S. Park, S.F. Ziegler, D.E. Williams, C.B. Ware, J.D. Meyer, and B.L. Davison. 1994. Early lymphocyte expansion is severely impaired in interleukin 7 receptor-deficient mice. I. Exp. Med. 180: 1955-1960.

Robey, E., D. Chang, A. Itano, D. Cado, H. Alexander, D. Lans, G. Weinmaster, and P. Salmon. 1996. An activated form of Notch influences the choice between CD4 and CD8 T cell lineages. Cell 87: 483-492.

Rolink, A., D. Haasner, S.-I. Nishikawa, and F. Melchers.1993. Changes in frequencies of clonable pre B cells during life in different lymphoid organs of mice. Blood 81: 2290-2300.

Sasai, Y., R. Kageyama, Y. Tagawa, R. Shigemoto, and S. Nakanishi. 1992. Two mammalian helix-loop-helix factors structurally related to Drosophila hairy and Enhancer of split. Genes \& Dev. 6: 2620-2634.

Shinkai, Y., G. Rathbun, K.-P. Lam, E.M. Oltz, V. Stewart, M. Mendelsohn, J. Charron, M. Datta, F. Young, A.M. Stall, and F.W. Alt. 1992. Rag-2-deficient mice lack mature lymphocytes owning to inability to initiate $\mathrm{V}(\mathrm{D}) \mathrm{J}$ rearrangement. Cell 68: 855-867.

Shortman, K. and L. Wu. 1996. Early T lymphocyte progenitors. Annu. Rev. Immunol. 14: 29-47.

Ström, A., P. Castella, J. Rockwood, J. Wagner, and M. Caudy. 1997. Mediation of NGF signaling by post-translational inhibition of HES-1, a basic helix-loop-helix repressor of neuronal differentiation. Genes \& Dev. 11:3168-3181.

Sudo, T., M. Ito, Y. Ogawa, M. Iizuka, H. Kodama, T. Kunisada, S.-I. Hayashi, M. Ogawa, K. Sakai, S. Nishikawa, and S.-I. Nishikawa. 1989. Interleukin 7 production and function in stromal cell-dependent B cell development. J. Exp. Med. 170: 333-338.

Ting, C.-N., M.C. Olson, K.P. Barton, and J.M. Leiden. 1996. Transcription factor GATA-3 is required for development of the T-cell lineage. Nature 384: 474-478.

Tomita, K., M. Ishibashi, K. Nakahara, S.-L. Ang, S. Nakanishi, F. Guillemot, and R. Kageyama. 1996. Mammalian hairy and Enhancer of split homolog 1 regulates differentiation of retinal neurons and is essential for eye morphogenesis. Neuron 16: 723-734.

von Boehmer, H. and H.J. Fehling. 1997. Structure and function of the pre-T cell receptor. Annu. Rev. Immunol. 15: 433452 . 


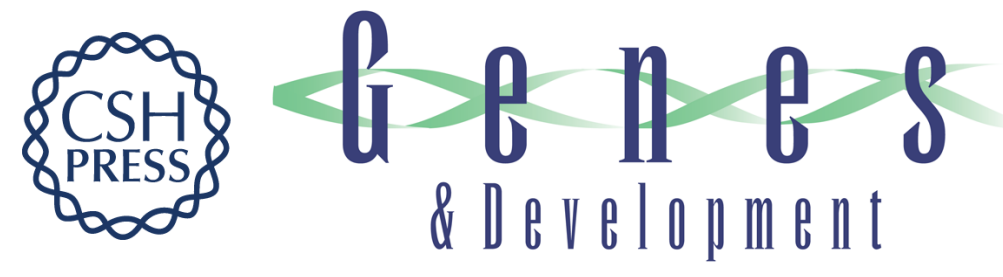

\section{The bHLH gene Hes1 is essential for expansion of early $\mathrm{T}$ cell precursors}

Koichi Tomita, Masakazu Hattori, Eijiro Nakamura, et al.

Genes Dev. 1999, 13:

References This article cites 29 articles, 12 of which can be accessed free at:

http://genesdev.cshlp.org/content/13/9/1203.full.html\#ref-list-1

License

Email Alerting Receive free email alerts when new articles cite this article - sign up in the box at the top Service right corner of the article or click here.

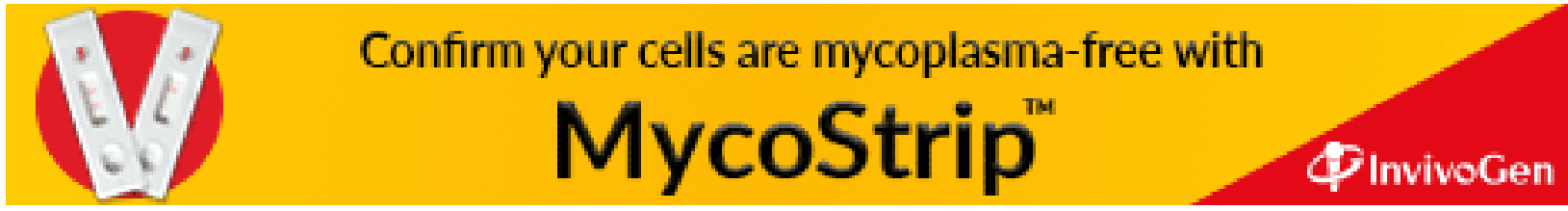

